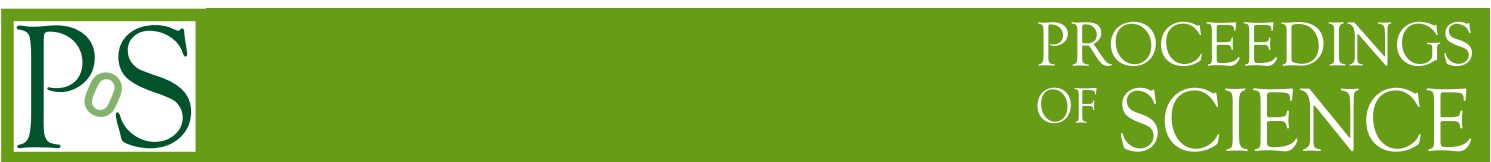

\title{
Charmonium Potentials at Finite Temperature
}

\author{
Chris Allton* \\ Swansea University \\ E-mail: c.alltoneswan.ac.uk

\section{Wynne Evans} \\ Swansea University \\ E-mail: pyevanseswan.ac.uk

\section{Jon-Ivar Skullerud} \\ National University of Ireland Maynooth \\ E-mail: jonivar@thphys.nuim.ie
}

\begin{abstract}
The charmonium states at non-zero temperature are studied on anisotropic lattices with 2 dynamical quark flavours. Non-local operators are used to determine the Nambu-Bethe-Salpeter (NBS) wavefunctions via both conventional fitting methods and the Maximum Entropy Method. The interquark potential is determined from the solution of the Schrodinger equation, given the NBS wavefunction as input following the HAL QCD method. We observe a temperature dependent potential which becomes steeper as the temperature decreases.
\end{abstract}

The 30th International Symposium on Lattice Field Theory - Lattice 2012

June 24-29, 2012

Cairns, Australia

*Speaker. 


\section{Introduction}

The quark-gluon plasma phase of QCD has been studied experimentally at both RHIC and the LHC. However, a full theoretical understanding of this phase is still being developed. One quantity which can aid this understanding is the potential between quarks as a function of temperature. Due to the widely cited $\mathrm{J} / \psi$ suppression [1], it is natural to consider the interquark potential of the charmonium system.

There has been theoretical work studying the interquark potential in quarkonia as a function of temperature from early models [2] to perturbative QCD calculations [3]. Furthermore, there have been some recent non-perturbative QCD studies (i.e. using lattice simulations) of interquark potentials which is relevant to the work presented here. These fall into two categories: (i) nonzero temperature studies of the static quark potential [4]; and (ii) zero temperature studies of the potential between quarks with finite masses [5]. This work presents a study of the interquark potential of charmonium using physical charm quark masses at finite temperature and uses twoflavours of dynamical quarks. A particular feature of our work is that our lattices are anisotropic which has the significant advantage in that our correlation function data is determined at a large number of temporal points.

Our method follows the HAL QCD method [6] of determining the potential in lattice simulations. ${ }^{1}$ This is based on using the Nambu-Bethe-Salpeter (NBS) wavefunction as input into the Schrödinger equation and solving for the potential.

In this work we use two methods to determine the wavefunction from our lattice simulations, conventional exponential fits and the Maximum Entropy Method. We find that both approaches give qualitatively similar results. Our main conclusion is that we observe a temperature dependence in the charmonium potential which is consistent with expectations, i.e. the potential is steepest for low temperatures, $T$, and shows signs of flattening at large distances as $T$ increases.

We are in the process of extending our work by using the "time-dependent" approach of HAL QCD [8] which determines the potential directly from the hadron correlation functions [9].

\section{Schrödinger Equation Approach}

We follow the HAL QCD method to determine the potential [6]. We begin by determining the NBS wavefunction of charmonium $\psi \underset{\sim}{r}$ ) from the $(t \rightarrow \infty$ behaviour of the $)$ correlators of pointsplit operators, $J(x, \underset{\sim}{r})=q(x) \Gamma U(x, x+\underset{\sim}{r}) \bar{q}(x+\underset{\sim}{r})$,

$$
C(\underset{\sim}{r}, t)=\underset{\sim}{\underset{\sim}{x}}<J(0 ; \underset{\sim}{r}) J(x ; \underset{\sim}{r})>\longrightarrow|\psi \underset{\sim}{r}|^{2} e^{-E t} .
$$

The NBS wavefunction is determined from the matrix element of the point-split operator, $\psi(\underset{\sim}{r}) \sim$ $\langle 0|J(\underset{\sim}{r})|$ gnd $\rangle$. Once the NBS wavefunctions and energies, $E$, are extracted from the correlators (see section 4) we use the Schrödinger equation to solve for the potential $V(r)$,

\footnotetext{
${ }^{1}$ The original HAL QCD programme studied internucleon rather than interquark potentials from lattice QCD simulations.
} 


$$
\left(-\frac{\nabla^{2}}{2 \mu}+V(r)\right) \psi(r)=E \psi(r)
$$

Here, $\mu=\frac{1}{2} m_{q} \simeq \frac{1}{4} M_{J / \psi}$, is the reduced mass. We note that this is the opposite approach normally associated with the Schrödinger equation, i.e. the potential is "reverse engineered" from the inputted wavefunction.

\section{Lattice Parameters and Correlators}

Our lattices are generated with two dynamical flavours of light quarks using a Wilson-type action with anisotropy of $\xi=a_{s} / a_{\tau}=6$ with $a_{s} \simeq 0.162 \mathrm{fm}$ and $a_{\tau}^{-1} \simeq 7.35 \mathrm{GeV}$ [7]. Table 3 lists the lattice parameters used. We note that the range of temperatures, $T=1 /\left(a_{\tau} N_{\tau}\right)$, is from the confined phase up to $\sim 2 T_{c}$ where $T_{c}$ is the deconfining transition. The charm quark is simulated with the (anisotropic) clover action and its mass is set by matching the experimental $\eta_{c}$ mass at zero temperature.

\begin{tabular}{ccccr}
\hline$N_{s}$ & $N_{\tau}$ & $T(\mathrm{MeV})$ & $T / T_{c}$ & \multicolumn{1}{c}{$N_{\text {cfg }}$} \\
\hline 12 & 80 & 90 & 0.42 & 250 \\
12 & 32 & 230 & 1.05 & 1000 \\
12 & 28 & 263 & 1.20 & 1000 \\
12 & 24 & 306 & 1.40 & 500 \\
12 & 20 & 368 & 1.68 & 1000 \\
12 & 16 & 458 & 2.09 & 1000 \\
\hline
\end{tabular}

Table 1: Lattice parameters used, including spatial and temporal dimension, $N_{s}$ and $N_{\tau}$.

In Fig.1 we plot the pseudoscalar charmonium correlation functions for quark separations $r$ at various temperatures, see table 3 . Only on-axis separations, $\underset{\sim}{r}$, were studied in this work.

\section{Wavefunctions and Potentials from Exponential Fits}

We extract the NBS wavefunction, $\psi(r)$, discussed in section 2 using a standard exponential fit of the point-split correlators at large $t, C(r, t)=|\psi(r)|^{2} e^{-E t}$ (see eq.(2.1)). The normalised wavefunctions are plotted in Fig.2 for both the pseudoscalar $\left(\eta_{c}\right)$ and vector $(J / \psi)$ channels. As can be seen, the expected behaviour for s-wave ground states is observed with the maximum of $\psi(r)$ at $r=0$, and $\psi(r) \rightarrow 0$ as $r \rightarrow 0$.

We use eq.(2.2) to determine the potential for both the pseudoscalar and vector channels. Figure 3 shows the spin-independent potential, $V_{q \bar{q}}(r)$, defined

$$
V_{q \bar{q}}(r)=\frac{1}{4}\left[V_{\mathrm{PS}}(r)+3 V_{V}(r)\right] .
$$

As can be seen from Fig. 3, there is evidence of a temperature dependency in the potential: the potential flattens as the temperature increases. This is in accord with expectations. Note however, that the $N_{t}=16$ correlator fits cannot be made over a large time range due to the brevity of the 

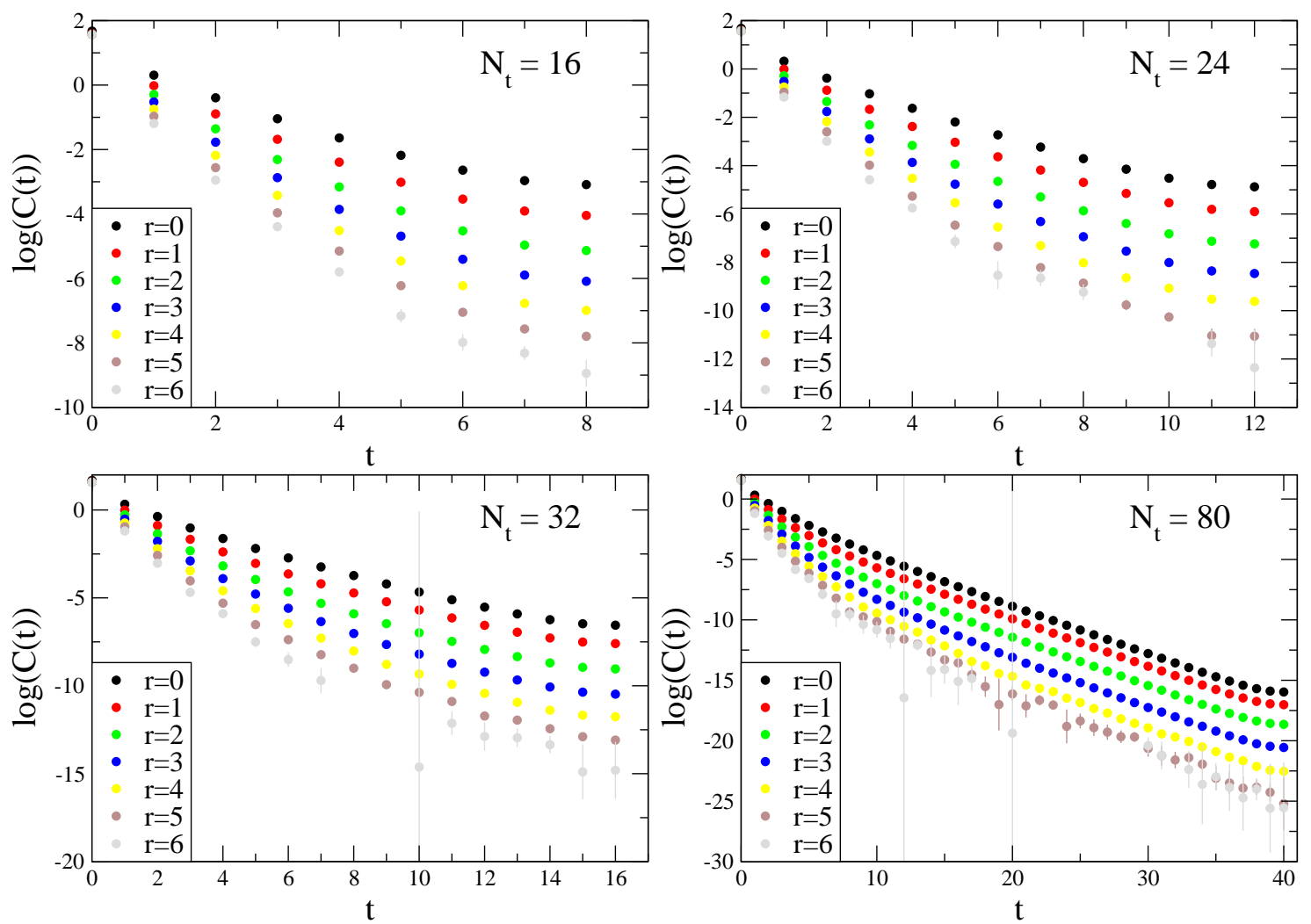

Figure 1: Pseudoscalar charmonium correlators of point-split operators for various temperatures.

lattice in the temporal direction. For this reason we associate some uncertainty with this ensemble (and these points are depicted with open symbols in Fig. 3).

\section{Wavefunctions from MEM}

The Maximum Entropy Method (MEM) has been used by many lattice studies to extract spectral information from correlators [10]. The fundamental equation is

$$
C(r, t)=\int \rho(r, \omega) K(t, \omega) d \omega,
$$

where $\rho$ is the spectral function and the lattice kernel is

$$
K(t, \omega)=\frac{\cosh \left[\omega\left(t-N_{t} / 2\right)\right]}{\sinh \left[\omega N_{\tau} / 2\right]} .
$$

Figure 4 shows the spectral function obtained via MEM from the correlators of point-split operators, $C(r, t)$, at the highest temperature. The vertical bands in Fig. 4 are placed at the ground and first excited states' masses as obtained from an MEM analysis at lower temperatures. We estimate the NBS wavefunction from the integral of $\rho(\omega)$ over this energy interval. Figure 2 includes the wavefunctions obtained in this way. As can be seen there is reasonable agreement between the MEM determination and the exponential fit approach from section 4. 

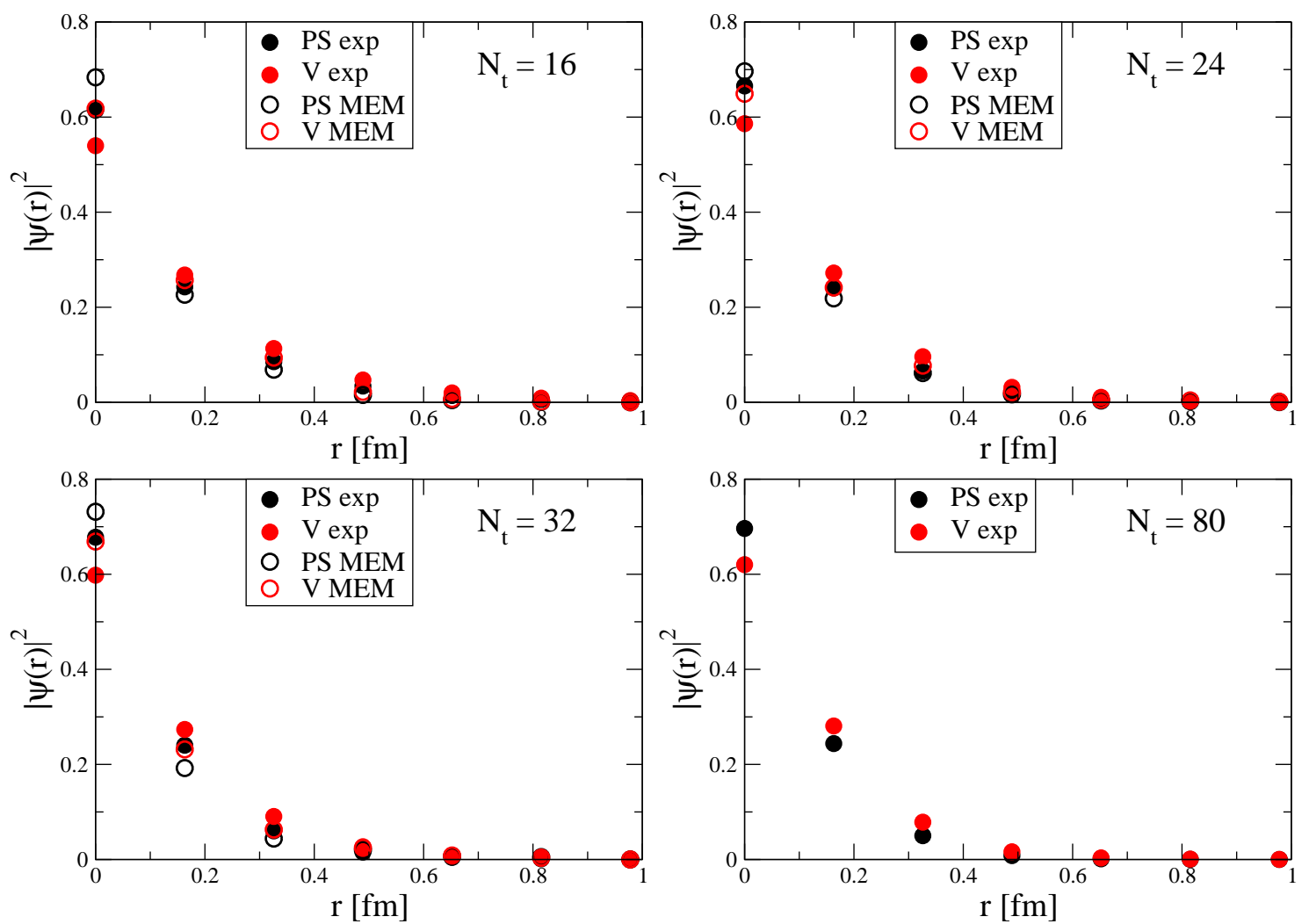

Figure 2: Pseudoscalar (PS) and vector (V) normalised wavefunctions, $\psi(r)$, for various temperatures. Both the results from the exponential and MEM fits are shown.

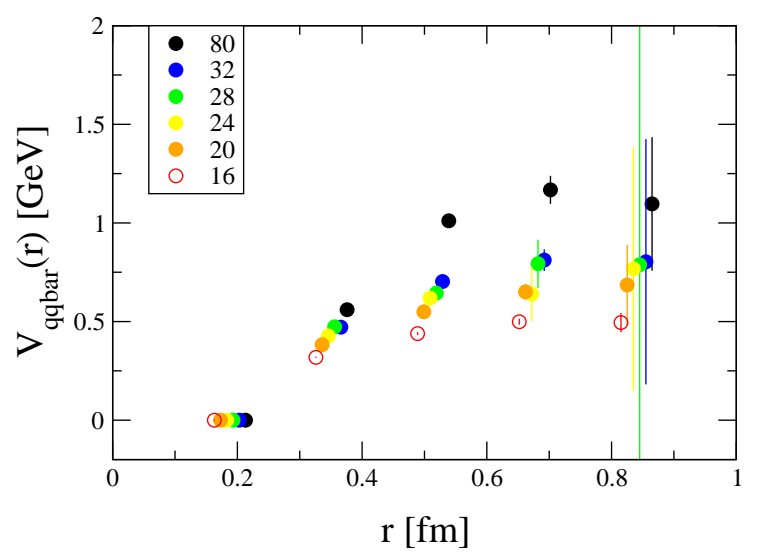

Figure 3: The spin-independent potential, $V_{q \bar{q}}$, for various temperatures. Statistical errors only are shown, and the points are shifted horizontally for clarity. A vertical constant was added to each temperature's potential to align the first point, i.e. $V_{q \bar{q}}\left(r / a_{s}=1\right)$ is defined to be zero. The $N_{t}=16$ points are depicted with open symbols due to the concerns about their fits as discussed in the text. 


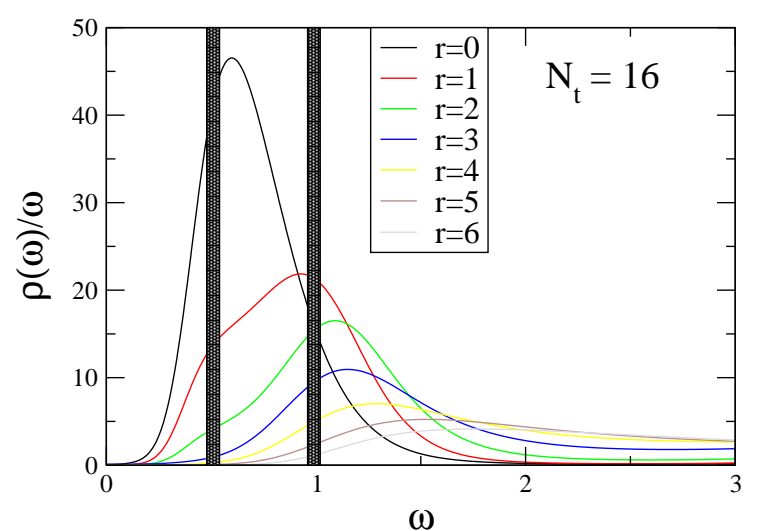

Figure 4: The spectral function obtained from MEM for the $N_{\tau}=16$ case for various separations $r$. The vertical bands are placed at the position of the ground and excited states.

Note that the estimate of first excited state's wavefunction from the second vertical band in Fig. 4 decreases for small separations, $r$, as $r \rightarrow 0$, as expected for an excited state.

\section{Conclusions and Outlook}

There is a significant body of theoretical work studying the interquark potential in charmonium at non-zero temperature using model, perturbative and lattice (non-perturbative) approaches. This work improves upon earlier lattice simulations by considering quarks with finite mass, and thus represents a first-principle calculation of the charmonium potential of QCD at finite temperature. The method we use is based on the HAL QCD approach which obtains the potential from correlators of point-split operators [6]. Our determination of the potential shows the expected flattening as the temperature increases. This work adds to previous charmonium studies performed by our collaboration with the same lattice parameters [11].

We've used two different methods of fitting the point-split correlators: conventional fits to exponentials and the MEM. There is qualitative agreement between the wavefunctions determined from both. However, it is clear from Fig 4 that the (high temperature) correlators, are not a simple sum of discrete exponentials, i.e. each spectral feature has a finite width. For this reason, fitting the correlator to an exponential (as in section 4) is an approximation. A better approach would be to determine the wavefunction from MEM as described in section 5. However, it is necessary to have symmetric correlators ${ }^{2}$ when using MEM to maintain positive semi-definite spectral weights, $\rho$. Since the operators in question in this study are point-split and therefore are inherently noisy, demanding symmetric correlators increases the noise substantially compared to non-symmetric correlators.

In forthcoming work [9] we will use the HAL QCD "time dependent" approach [8] which allows us to calculate the potential directly from the correlators, thus circumventing the problems discussed in both the exponential and MEM fitting procedures. We also will be studying signif-

\footnotetext{
${ }^{2}$ i.e. correlators of the same operator at the source and sink
} 
icantly larger lattices (with a spatial volume of $32^{3}$ ) with $2+1$ flavours of dynamical quarks and hope to extend our work to determine the potential between NRQCD quarks [12].

\section{Acknowledgements}

We acknowledge the support and infrastructure provided by the Trinity Centre for High Performance Computing and the IITAC project funded by the HEA under the Program for Research in Third Level Institutes (PRTLI) co-funded by the Irish Government and the European Union. The work of CA and GA is carried as part of the UKQCD collaboration and the DiRAC Facility jointly funded by STFC, the Large Facilities Capital Fund of BIS and Swansea University. WE and CA are supported by STFC. CRA thanks the Galileo Galilei Institute for Theoretical Physics for hospitality and the INFN for support during the writing up of this work. We are very grateful to Sinya Aoki, Balint Joó and Robert Edwards for useful discussions.

\section{References}

[1] T. Matsui and H. Satz, Phys. Lett. B 178 (1986) 416.

[2] F. Karsch, M. T. Mehr and H. Satz, Z. Phys. C 37 (1988) 617.

[3] Y. Burnier, M. Laine and M. Vepsalainen, JHEP 0801 (2008) 043 [arXiv:0711.1743 [hep-ph]], N. Brambilla, J. Ghiglieri, A. Vairo and P. Petreczky, Phys. Rev. D 78 (2008) 014017 [arXiv:0804.0993 [hep-ph]], A. Dumitru, Y. Guo, A. Mocsy and M. Strickland, Phys. Rev. D 79 (2009) 054019 [arXiv:0901.1998 [hep-ph]].

[4] A. Rothkopf, T. Hatsuda and S. Sasaki, PoS LAT 2009 (2009) 162 [arXiv:0910.2321 [hep-lat]], Phys. Rev. Lett. 108 (2012) 162001 [arXiv:1108.1579 [hep-lat]], A. Rothkopf, arXiv:1207.5486 [hep-ph], Y. Burnier and A. Rothkopf, Phys. Rev. D 86 (2012) 051503 [arXiv:1208.1899 [hep-ph]]. A. Bazavov and P. Petreczky, arXiv:1210.6314 [hep-lat], P. Petreczky, C. Miao and A. Mocsy, Nucl. Phys. A 855 (2011) 125 [arXiv:1012.4433 [hep-ph]].

[5] T. Kawanai and S. Sasaki, Phys. Rev. D 85 (2012) 091503 [arXiv:1110.0888 [hep-lat], PoS LATTICE 2011 (2011) 126 [arXiv:1111.0256 [hep-lat]], Phys. Rev. Lett. 107 (2011) 091601 [arXiv:1102.3246 [hep-lat]], Y. Ikeda and H. Iida, PoS LATTICE 2010 (2010) 143 [arXiv:1011.2866 [hep-lat]], PoS LATTICE 2011 (2011) 195.

[6] N. Ishii, S. Aoki and T. Hatsuda, Phys. Rev. Lett. 99 (2007) 022001 [nucl-th/0611096], S. Aoki, T. Hatsuda and N. Ishii, Prog. Theor. Phys. 123 (2010) 89 [arXiv:0909.5585 [hep-lat]], S. Aoki et al. [HAL QCD Collaboration], arXiv:1206.5088 [hep-lat].

[7] M. B. Oktay and J. -I. Skullerud, arXiv:1005.1209 [hep-lat].

[8] N. Ishii [HAL QCD Collaboration], PoS LATTICE 2011 (2011) 160.

[9] W. Evans, C.R. Allton, J.-I. Skullerud, in preparation.

[10] M. Asakawa, T. Hatsuda and Y. Nakahara, Prog. Part. Nucl. Phys. 46 (2001) 459 [hep-lat/0011040].

[11] G. Aarts, C. Allton, M. B. Oktay, M. Peardon and J. -I. Skullerud, Phys. Rev. D 76 (2007) 094513 [arXiv:0705.2198 [hep-lat]].

[12] G. Aarts, C. Allton, S. Kim, M. P. Lombardo, M. B. Oktay, S. M. Ryan, D. K. Sinclair and J. -I. Skullerud, arXiv:1210.2903 [hep-lat]. 